This item was submitted to Loughborough's Research Repository by the author.

Items in Figshare are protected by copyright, with all rights reserved, unless otherwise indicated.

\title{
How does Oyster work? The simple interpretation of Oyster mathematics
}

PLEASE CITE THE PUBLISHED VERSION

http://dx.doi.org/10.1016/j.euromechflu.2014.03.007

\section{PUBLISHER}

(C) Elsevier Masson SAS

\section{VERSION}

AM (Accepted Manuscript)

\section{PUBLISHER STATEMENT}

This work is made available according to the conditions of the Creative Commons Attribution-NonCommercialNoDerivatives 4.0 International (CC BY-NC-ND 4.0) licence. Full details of this licence are available at: https://creativecommons.org/licenses/by-nc-nd/4.0/

\section{LICENCE}

CC BY-NC-ND 4.0

\section{REPOSITORY RECORD}

Renzi, Emiliano, K. Doherty, A. Henry, and F. Dias. 2019. "How Does Oyster Work? the Simple Interpretation of Oyster Mathematics". figshare. https://hdl.handle.net/2134/17136. 


\title{
How does Oyster work? The simple interpretation of Oyster mathematics
}

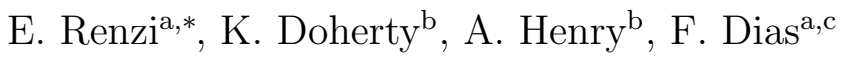 \\ ${ }^{a}$ UCD School of Mathematical Sciences, University College Dublin, Belfield Dublin 4, \\ Ireland. \\ ${ }^{b}$ Aquamarine Power Limited, Elder House, 24 Elder Street Edinburgh EH1 3DX, UK. \\ ${ }^{c}$ Centre de Mathématiques et de Leurs Applications (CMLA), Ecole Normale Supérieure \\ de Cachan, 94235 Cachan, France
}

\begin{abstract}
Oyster ${ }^{\circledR}$ is a surface-piercing flap-type device designed to harvest wave energy in the nearshore environment. Established mathematical theories of wave energy conversion, such as 3D point-absorber and 2D terminator theory, are inadequate to accurately describe the behaviour of Oyster, historically resulting in distorted conclusions regarding the potential of such a concept to harness the power of ocean waves. Accurately reproducing the dynamics of Oyster requires the introduction of a new reference mathematical model, the "flap-type absorber". A flap-type absorber is a large thin device which extracts energy by pitching about an horizontal axis parallel to the ocean bottom. This paper unravels the mathematics of Oyster as a flap-type absorber. The main goals of this work are to provide a simple - yet accurate - physical interpretation of the laws governing the mechanism of wave power absorption by Oyster and to emphasise why some other, more established, mathematical theories cannot be expected to accurately describe its behaviour.
\end{abstract}

Keywords:

Wave energy, Oyster, fluid-structure interactions, applied mathematics

\footnotetext{
${ }^{*}$ Corresponding author

Email address: emiliano.renzi@ucd.ie (E. Renzi)
} 


\section{Introduction}

Wave-structure interaction is a fundamental subject in Ocean Engineering and has been one of Enok Palm's most favoured areas of research [1, 2, 3, 4]. In recent years, wave-structure interaction has attracted renewed attention by the scientific community, due to the growing interest in energy extraction from ocean waves and in the design of wave energy converters (WECs).

Early work on WECs was focused mainly towards floating devices like point and line absorbers [5]. A point absorber is a device whose dimensions are much smaller than the incident wavelength (e.g. a heaving buoy), while a line absorber has one dominant horizontal dimension, whose order of magnitude is at least one wavelength (e.g. an articulated raft) [6]. Line absorbers can work either as terminators or attenuators, depending on their alignment being, respectively, orthogonal or parallel to the direction of propagation of the incident waves [5]. However, driven by the need of making more powerful WECs to decrease energy production costs and increase competitiveness against fossil fuels, lately the wave energy sector has evolved towards the design of new large-scale WECs which do not belong to the point- and lineabsorber categories $[5,7,8]$.

Oyster $^{\circledR}$ is a surface-piercing flap-type device designed by Aquamarine Power Limited to harvest ocean energy in the nearshore and convert it into clean electricity, as shown in figure 1. According to a modern technologybased WEC classification (see for example www.aquaret.com), Oyster belongs to the category of oscillating wave surge converters (OWSCs). OWSCs are bottom-hinged oscillators which essentially pitch as inverted pendulums, following the surge movement of the water particles in the nearshore [7]. Appropriate power take-off (PTO) systems linked to the device allow extraction of useful energy. For Oyster, a set of pistons are activated by the oscillating movement of the flap. The pistons pump high-pressure water to drive an onshore hydro-electric turbine, from which electricity is conventionally generated (see again figure 1). In recent years, the Oyster concept has proved to be very promising and is leading the way towards the large-scale exploitation of the ocean energy resource. The first full-scale $315 \mathrm{~kW}$ Oyster proof-of-concept device was successfully installed and operated off the coast of Orkney (Scotland, U.K.) in 2009. In June 2012 operational testing of the second-generation device Oyster 800 commenced at the European Marine Energy Centre (EMEC) in Orkney, delivering electrical power to the national grid. Oyster 800 measures $26 \mathrm{~m}$ across its width, is installed 


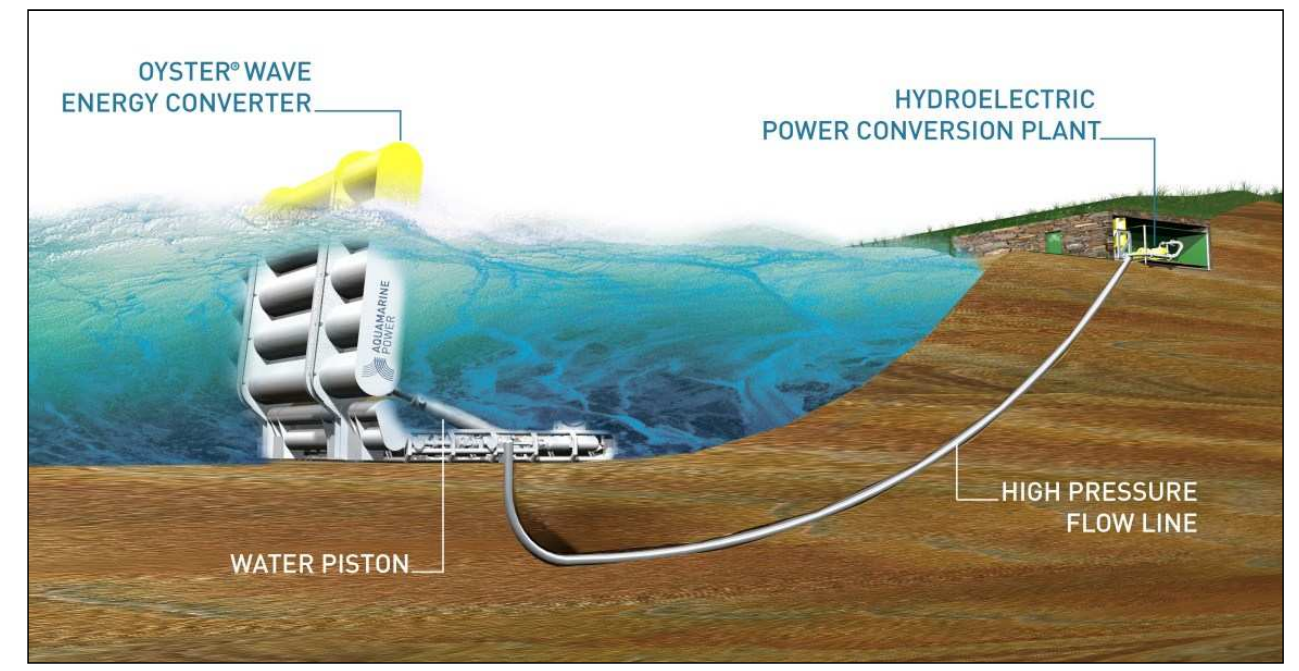

Figure 1: Artist's sketch of the Oyster WEC concept (online figure in colour).

at a depth of $13 \mathrm{~m}$, at approximately $500 \mathrm{~m}$ from the shore. With a maximum generating capacity of $800 \mathrm{~kW}$, Oyster 800 recently generated $1 \mathrm{MWh}$ in 5 hours on a single power cylinder, which is believed to be the highest sustained power output of any wave energy machine in the world (source: www.aquamarinepower.com).

How does Oyster work? What makes the Oyster concept successful? In this paper we will unravel the Oyster hydrodynamics, based on new semianalytical models recently developed at University College Dublin under the research project "High-end computational models for wave energy systems" funded by Science Foundation Ireland (SFI), in collaboration with Aquamarine Power $[9,10,11,12,13,14,15]$. The main aim of this paper is to provide a simple - yet accurate - physical interpretation of the mathematics governing Oyster's operating principles, which are substantially different from those governing the behaviour of point and two-dimensional line absorbers. We hope that this work will be helpful to the broader scientific and engineering audience and to the even wider community of wave energy enthusiasts.

In Section 2 we shall quickly review the hydrodynamic problem, introducing the basic physical laws which govern the motion of Oyster in monochromatic waves. This will allow us to address a simple yet fundamental question in Section 3: why does Oyster pitch? The answer will clearly rule out the 
applicability of the point-absorber theory to describe Oyster's dynamics. In Section 4 we shall further analyse the mechanism of energy extraction from Oyster. We will show that Oyster reaches high levels of efficiency without the need of tuning it to resonance with the incident waves. In particular, we will prove that Oyster's capture factor (a non-dimensional parameter commonly used to measure WECs' efficiency) can be larger than the theoretical maximum value predicted by the classical two-dimensional (2D) theory for terminator-type devices $[6,16]$. In the concluding Section, we will introduce the flap-absorber concept, fixing the lack of an appropriate mathematical framework for oscillating wave surge converters.

\section{How does Oyster work?}

\subsection{Governing physical laws}

Oyster is driven by ocean waves. As such, it obeys the physical laws governing the dynamics of floating bodies in fluids. Wave-body interaction usually occurs without sensitive variation in water density, so that the fluid can be practically assumed to be incompressible for the whole duration of the process [16]. Furthermore, given the characteristic wave amplitude $A$ and the characteristic body dimension $w$, the fluid can be represented as inviscid when $A \ll w$, as shown by [16]. For Oyster 800, the typical wave amplitude in operational conditions is of the order $A \simeq 1 \mathrm{~m}$ [7], while the characteristic dimension is the width $w=26 \mathrm{~m}$, so that $A / w \simeq 0.03 \ll 1$. Hence an inviscid theory can be safely adopted to model the hydrodynamics of Oyster in operational seas. Finally, in such conditions the vorticity of the flow field can be neglected and the flow can be considered irrotational (see again [16]). Incidentally, note that in exceptionally energetic sea states, for which $A / w<1$ but not $A / w \ll 1$, the present approximation is no longer valid and alternative research techniques must be employed to capture the viscous vortex dynamics arising in large-amplitude, highly nonlinear motions. One such alternative is bespoke computational fluid dynamic (CFD) models such as those presented in $[17,18,19,20]$.

In the case $A / w \ll 1$, assuming irrotational flow of inviscid fluid, the velocity of the flow field can be expressed as $\mathbf{u}=\nabla \Phi$, where $\nabla=(\partial / \partial x, \partial / \partial y$, $\partial / \partial z)$. $(x, y, z)$ determine a Cartesian reference system with the $z$ axis pointing upwards from the mean sea level and the $(x, y)$ axes lying on the horizontal plane $z=0 . \Phi(x, y, z, t)$ is the unknown velocity potential and $t$ denotes time. The assumption $A / w \ll 1$ also implies that a linearised form of the 

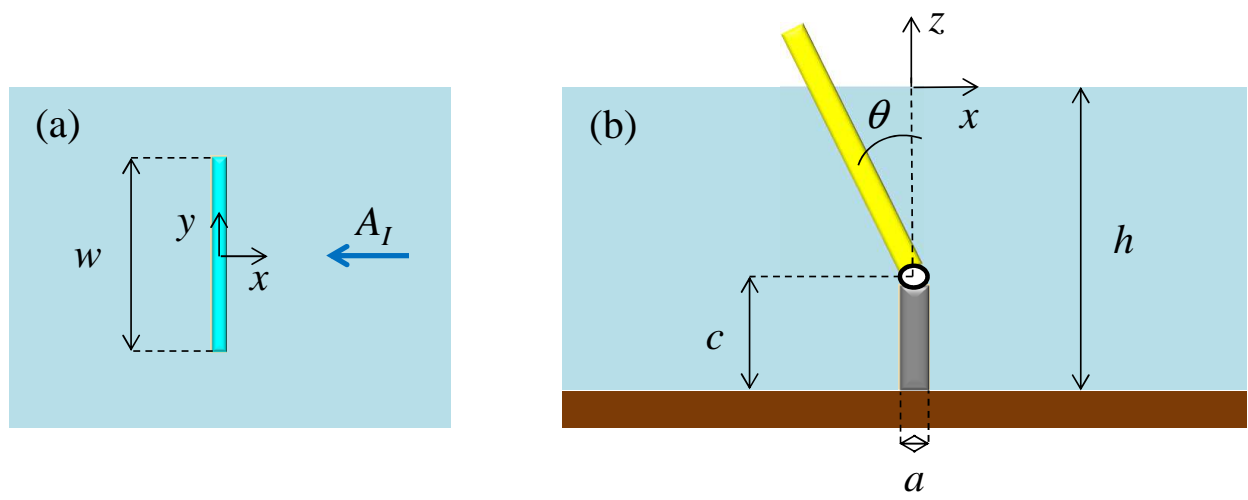

Figure 2: Geometry of the system; (a) plan view, (b) section (online figure in colour).

governing equations of the system can be used, as shown in [9]. Within this framework, the velocity potential must satisfy conservation of mass

$$
\nabla^{2} \Phi=0
$$

in the fluid domain and a linearised kinematic-dynamic boundary condition on the free surface

$$
\frac{\partial^{2} \Phi}{\partial t^{2}}+g \frac{\partial \Phi}{\partial z}=0, \quad z=0
$$

where $g$ is the acceleration due to gravity. Eq. (2) physically means that the particles on the free surface, subject to the atmospheric pressure, do not escape from the fluid. A no-flux condition at the bottom of the ocean, assumed impermeable and of constant depth $h$, is also required:

$$
\frac{\partial \Phi}{\partial z}=0, \quad z=-h
$$

together with an appropriate kinematic condition on the flap. The latter physically imposes that the motion of the water particles on the surface of the oscillating flap can be only tangential [16]; in other words, water particles cannot penetrate the flap. For simplicity, but without loss of physical meaning, here we shall represent Oyster as a rectangular flap of width $w$ hinged upon a foundation of height $c$, as depicted in figure 2. As already shown by [9], the flap thickness $a \ll w$ is immaterial for the solution of the potential-flow field (thin-plate approximation). With these assumptions, the kinematic condition on the flap reads

$$
\frac{\partial \Phi}{\partial x}=-\frac{\partial \theta(t)}{\partial t}(z+h-c) H(z+h-c), \quad x= \pm 0,|y|<w / 2,
$$


where $\theta(t)$ is the unknown angle of rotation and the Heaviside function $H$ ensures absence of normal flux through the foundation. Renzi and Dias $[9,10,11,12]$ first solved the boundary-value problem (1)-(4) with a careful application of the Green integral theorem in the fluid domain. The full mathematical treatise is reported in $[9,10,11,12]$, which the interested reader is addressed to for an in-depth understanding of the methods involved. In the following section, we shall report the semi-analytical solution of [11] in the open ocean and further highlight its physical meaning.

\subsection{Physical meaning of the mathematical solution}

The solution of the boundary-value problem (1)-(4) describes the waves generated by the fluid-structure interaction in terms of the velocity potential:

$$
\Phi=\Re\left\{\left[\phi_{I}(x, z)+\phi_{D}(x, y, z)+\phi_{R}(x, y, z)\right] e^{-i \omega t}\right\} .
$$

The latter expression physically represents oscillating waves of period $T=$ $2 \pi / \omega$, whose spatial variation is described by the sum of three different components, $\phi_{I}, \phi_{D}$ and $\phi_{R}$, respectively. The first term $\phi_{I}$ represents the incident wave field, which sets Oyster into motion, and is given by

$$
\phi_{I}=-\frac{i g A}{\omega} \frac{\cosh k(z+h)}{\cosh k h} e^{-i k x} .
$$

In the latter, $k$ is the wavenumber, solution of the well-known dispersion relation $\omega^{2}=g k \tanh k h$. Eq. (6) describes a monochromatic wave field of amplitude $A$ over a bottom of constant depth $h$. In this paper, only monochromatic incident waves will be considered to describe the fundamental physical behaviour of Oyster. Extension of this theory to random seas is detailed in [14]. Referring again to Eq. (5), $\phi_{D}$ is the diffraction potential, which describes the modification of the wave field induced by the physical presence of the flap held fixed in water. Mathematically, $\phi_{I}+\phi_{D}$ is the classical solution to the scattering problem, in which the flap is held fixed in incoming waves. Finally, $\phi_{R}$ is the radiation potential, which describes the wave field that in turn is generated by the flap once it is set into motion by the incident waves.

In summary, Eq. (5) physically means that, in response to the incident waves $\left(\phi_{I}\right)$, Oyster in turn generates waves $\left(\phi_{D}+\phi_{R}\right)$. Part of those waves $\left(\phi_{D}\right)$ are due to Oyster's obstructing reaction to the incident wave field, while part $\left(\phi_{R}\right)$ are further generated by Oyster's induced oscillating motion. $\phi_{D}$ 

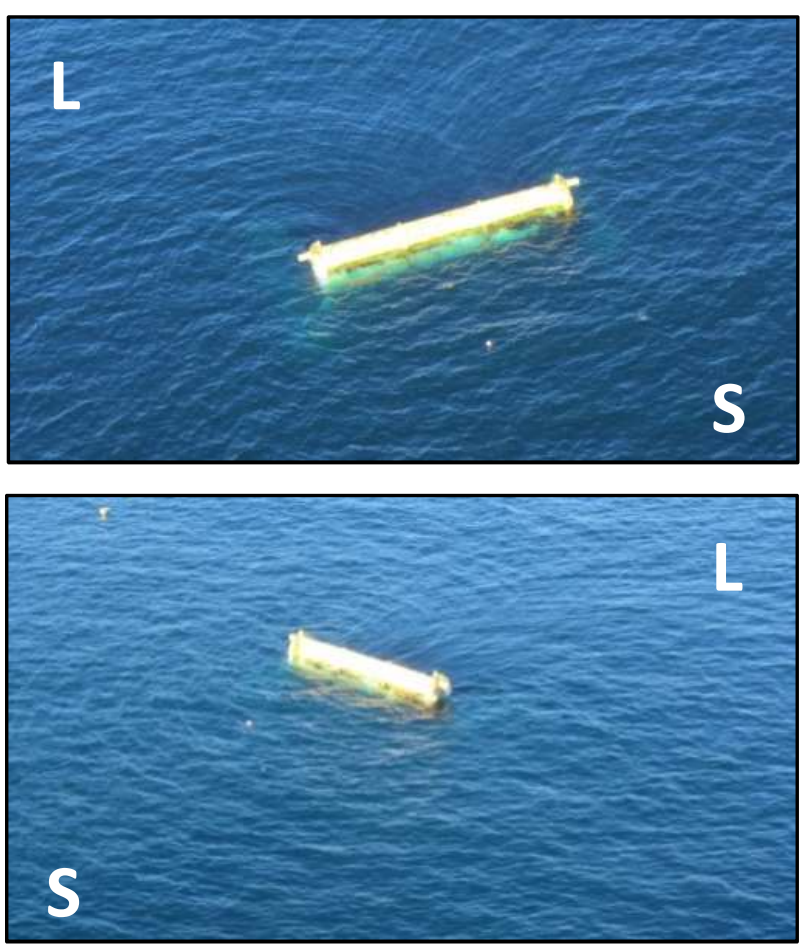

Figure 3: Patterns of outgoing wave radiation and diffraction by the Oyster 800 OWSC during operation in Orkney (U.K.). $\mathrm{S}=$ seaward side, $\mathrm{L}=$ landward side (online figure in colour).

and $\phi_{R}$ have complex mathematical expressions which are detailed in [11] and will not be reported here for the sake of brevity. Physically, both $\phi_{D}$ and $\phi_{R}$ represent waves propagating from the device into the open ocean, radially outgoing at large distance from the device [see 11, 12]. Patterns of radial propagation like those described by the mathematical expressions $\phi_{D}$ and $\phi_{R}$ of [11] have indeed been observed during the operation of Oyster 800 in Orkney, as shown for example in figure 3.

\section{Why does Oyster pitch?}

\subsection{Non- point-absorber dynamics}

Before Oyster, it was generally thought that a "good wave absorber must be a good wave-maker" [21]. Using this approach, the design effort is oriented towards optimising the radiating properties of the WEC to make it cancel out most of the incident ocean waves $\left(\phi_{I}\right)$ as they pass. This can be achieved by letting the WEC radiate a counteracting wave field $\left(\phi_{R}\right)$, in such a way 


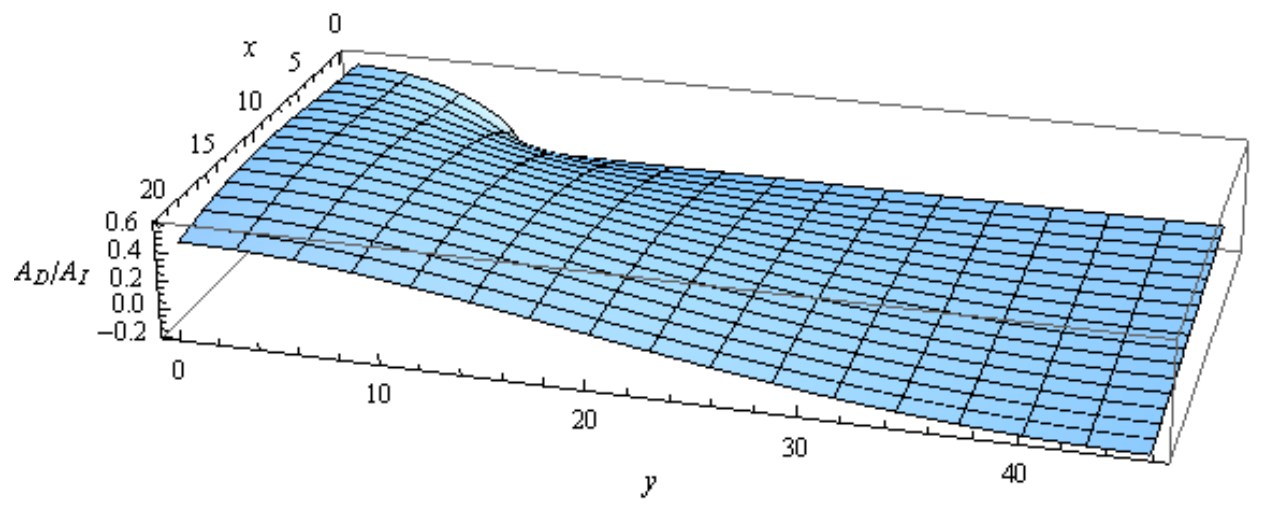

Figure 4: Snapshot of the ratio $A_{D} / A_{I}$ between the amplitude of the diffracted wave field $A_{D}$ (associated with $\phi_{D}$ ) and the amplitude of the incident wave $A_{I}$ in front of a fixed flap, under an incident wave crest. The flap (not represented) is located at $x=0$. Half the domain is represented because of symmetry about $y=0$. Parameters are $A=1 \mathrm{~m}$, $T=7 \mathrm{~s}, w=18 \mathrm{~m}, h=10.9 \mathrm{~m}$. The semi-analytical channel model of Renzi and Dias [9] has been used. The channel width is large enough $(92 \mathrm{~m})$ so that the lateral walls do not affect the near-field behaviour of the system [11] (online figure in colour).

that wave energy is absorbed by the WEC and little motion is transmitted behind it. Mathematically, this statement can be translated as:

$$
\Phi=\Re\left\{\left(\phi_{I}+\phi_{R}\right) e^{-i \omega t}\right\} \simeq 0
$$

down wave from the WEC [22]. This concept leads to small, resonant WECs with a focus on the radiated wave and its phase with the incident wave (see for example the incident/radiated wave patterns of the point-absorber device in figure 4 of [22]). Let us compare the general solution (5), which governs the hydrodynamics of any floating body in waves, with the mathematical point-absorber principle Eq. (7). It is immediate to notice that in Eq. (7) the diffracted wave field $\phi_{D}$ is neglected. This approximation proves indeed valid for WECs whose physical presence when held fixed in water induces little modification of the incident wave field, but is definitely not valid for a large device like Oyster [11]. As an example, figure 4 shows that the amplitude of the diffracted waves in front of a fixed flap is comparable $(O(1))$ to the amplitude of the incident waves. Clearly, for a flap-type converter like Oyster the diffraction dynamics associated with $\phi_{D}$ in Eq. (5) cannot be neglected. Indeed, in the following section we will show that Oyster's motion is triggered by diffractive, non- point-absorber dynamics. 


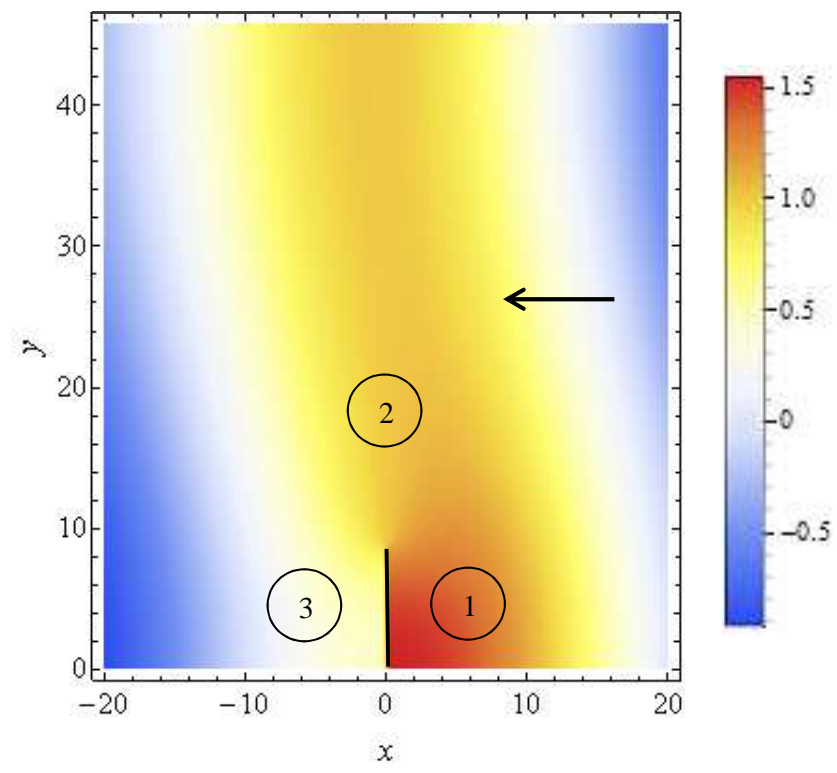

Figure 5: Density plot of the free-surface elevation in physical variables around a fixed flap for an incident wave crest. The arrow indicates the direction of the incoming waves. Half the flap is represented because of symmetry about the horizontal line $y=0$. Values are in metres. Parameters are $A=1 \mathrm{~m}, T=7 \mathrm{~s}, w=18 \mathrm{~m}, h=10.9 \mathrm{~m}$. The semianalytical model of Renzi and Dias [9] has been used. Labels indicate: 1 - reflection zone, 2 - refraction (bending) zone, 3 - shading zone (online figure in colour).

\subsection{Oyster's exciting dynamics}

The fundamental dynamics of Oyster can be easily understood by first looking at the modification that it induces in the wave field when held fixed in incoming waves. Figure 5 shows the incident-diffracted wave $\left(\phi_{I}+\phi_{D}\right)$ pattern on a region close to a fixed flap, when the latter encounters the wave crest. The incident waves are partially obstructed by the flap and the freesurface elevation is maximum in front of it, where the reflected wave sums up to the incident wave (zone 1). Laterally, crests bend to overcome the obstacle, showing a three-dimensional refractive behaviour (zone 2). In the meantime, the shading effect of the flap attenuates the wave amplitude at the lee side (zone 3). Reflecting, bending and shading the incident wave field are diffractive properties of the flap. As a result of this complex three-dimensional dynamics, a strong difference in free-surface elevation generates between the sides of the flap (see again figure 5), accompanied by a difference in dynamic pressure. Figure 6 shows the contour plot of the pressure difference between the two sides of the flap under the action of the waves represented in figure 5. The pressure difference is maximum at the centre (top left of figure 6) and 


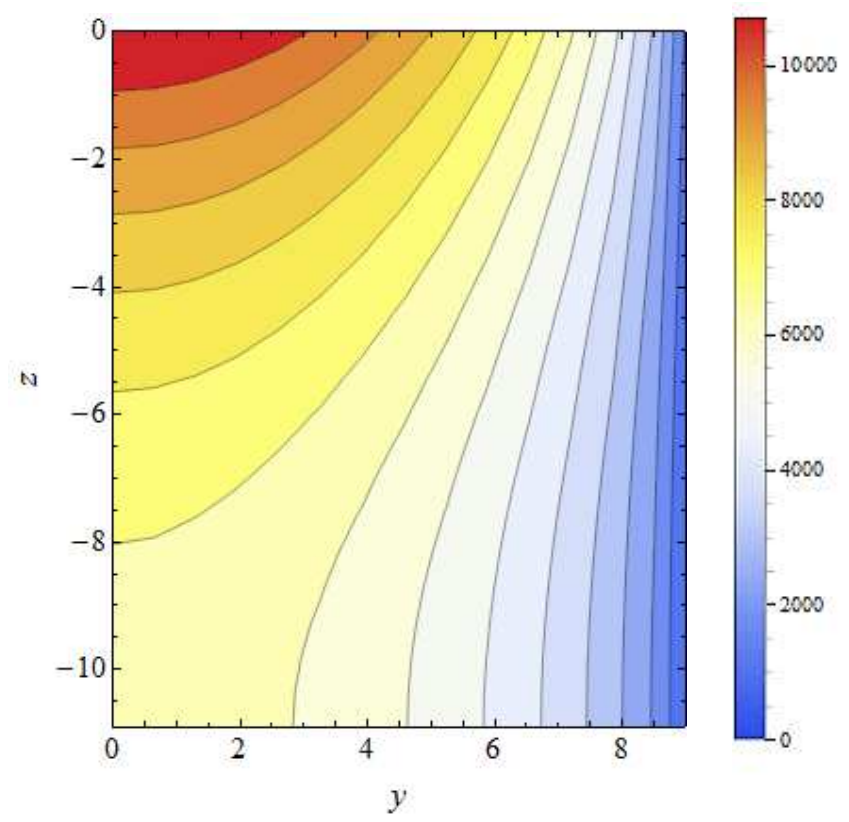

Figure 6: Contour plot of the pressure difference $(\mathrm{Pa})$ on a fixed flap for an incident wave crest. Half the flap is represented because of symmetry. Values of $(y, z)$ are in metres. Parameters are $A=1 \mathrm{~m}, T=7 \mathrm{~s}, w=18 \mathrm{~m}, h=10.9 \mathrm{~m}$. The semi-analytical model of Renzi and Dias $[9,10,11]$ has been used (online figure in colour).

then decays towards the lateral edge due to continuity. Such net pressure distribution on the flap generates an exciting torque about the hinge axis (see figure $2 \mathrm{~b}$ ). If the flap in figure 5 is now left free to oscillate, it would pitch towards the left under the action of the exciting torque.

In conclusion, Oyster pitches driven by the strong exciting torque resulting from the pressure difference between its sides. Such pressure difference originates because of Oyster's ability to favourably reflect, bend and shade waves in different areas of the surrounding sea. This diffractive dynamics is much stronger than that occurring with point absorbers and therefore has required a non- point-absorber explanation.

\section{How does Oyster extract energy from the ocean?}

\subsection{Mechanism of energy extraction}

As anticipated in Section 1, Oyster extracts energy by means of a PTO system resisting the motion of the body. In mechanical terms, Oyster is a forced damped oscillator. The forcing term is indeed the hydrodynamic 
torque resulting from the waves pushing Oyster's surface. The damping action of the PTO system is instead modelled as a resisting torque proportional to the angular velocity of the flap. Mathematically, Oyster's law of motion in the frequency domain is (see [11])

$$
\left[-\omega^{2}(I+\mu)+C-i \omega\left(\nu+\nu_{p t o}\right)\right] \Theta=F_{D}
$$

where $\Theta$ is the unknown complex amplitude of rotation and $i$ the imaginary unit. Physically, Eq. (8) represents the dynamic equilibrium between the following actions:

- $F_{D}$ is the complex exciting torque, resulting from the dynamic pressure jump in the scattering problem (see e.g. figure 6).

- $\omega^{2} I \Theta$ represents the inertial action, $I$ being the moment of inertia of the flap with respect to the hinge axis.

- $\omega^{2} \mu \Theta$ indicates the added inertial action. $\mu$ is the added inertia, associated with the hydrodynamic inertia of the water particles surrounding the flap.

- $C \Theta$ represents the buoyancy restoring action, $C$ being the buoyancy torque acting on the flap per unit amplitude of rotation (pitch stiffness).

- $\omega\left(\nu+\nu_{p t o}\right) \Theta$ represents the total damping action. $\nu_{p t o}$ is the PTO damping, while $\nu$ is the radiation damping. The latter is related to the energy radiated away by the pitching flap, which is indeed a source of damping for the system.

As shown in [11], $F_{D}$ is determined from the diffraction potential $\phi_{D}$, while $\mu$ and $\nu$ are obtained from the radiation potential $\phi_{R}$. I and $C$ depend on the design layout of the flap and $\nu_{p t o}$ depends on the PTO strategy. Note that in (8) the buoyancy torque acting on the flap is fully accounted for. In other words, the thin-plate approximation is applied only to the velocity potential (5) and does not affect the buoyancy property of the flap. The average power extracted over a cycle by Oyster is [7, 23]

$$
P=\frac{\left|F_{D}\right|^{2}}{4\left(\nu_{p t o}+\nu\right)}
$$


provided the PTO system is designed such that

$$
\nu_{p t o}=\sqrt{\frac{\left[C-(I+\mu) \omega^{2}\right]^{2}}{\omega^{2}}+\nu^{2}},
$$

which corresponds to the optimum PTO damping (see for example [21]). To assess the performance of Oyster, the capture factor (also known as capture width ratio, see [5])

$$
C_{F}=\frac{P}{\frac{1}{2} \rho g A_{I}^{2} C_{g} w}=\frac{\left|F_{D}\right|^{2}}{2 \rho g A_{I}^{2} C_{g} w\left(\nu_{p t o}+\nu\right)}
$$

is defined as the ratio between the power captured per unit Oyster width and the power available per unit crest length, where

$$
C_{g}=\frac{\omega}{2 k}\left(1+\frac{2 k h}{\sinh 2 k h}\right)
$$

is the group velocity of the incident wave. Note from Eq. (9) and Eq. (11) that both $P$ and $C_{F}$ are inversely proportional to the total damping and directly proportional to the square of the exciting torque. Hence Eq. (9) and Eq. (11) physically mean that Oyster's power capture $(P)$ and performance $\left(C_{F}\right)$ are dominated by the exciting torque, which is the power-generating action. This further clarifies the difference between Oyster and point-absorbers. While a point-absorber relies on its radiative properties to cancel out part of the incident waves [21, 22], Oyster relies on its diffractive properties to generate a large exciting action which amplifies the power capture $(9)[7,11,23]$. Therefore, an optimal design of Oyster must attempt to enhance the exciting torque on the device. As an example, figure 7 shows the plot of the exciting torque magnitude $\left|F_{D}\right|$ and capture factor $C_{F}$ for a device configuration similar to the Oyster 800 WEC developed by Aquamarine Power, with respect to the period of the incident wave. Note that the peak exciting torque of figure 7 (a) occurs at a short period, i.e. $T_{\text {peak }} \simeq 7 \mathrm{~s}$. Such design allows $T_{\text {peak }}$ to be close to the mean energy period $T_{e}$ of several suitable sites for Oyster deployment in the North Atlantic (see [24] and [25] for details). Note also that the capture factor curve of figure $7(\mathrm{~b})$ behaves very similarly to the exciting torque curve in figure 7(a). This further confirms that Oyster's power absorption and performance is driven by the exciting torque. An important consequence of this dynamics is that tuning Oyster to resonance is 

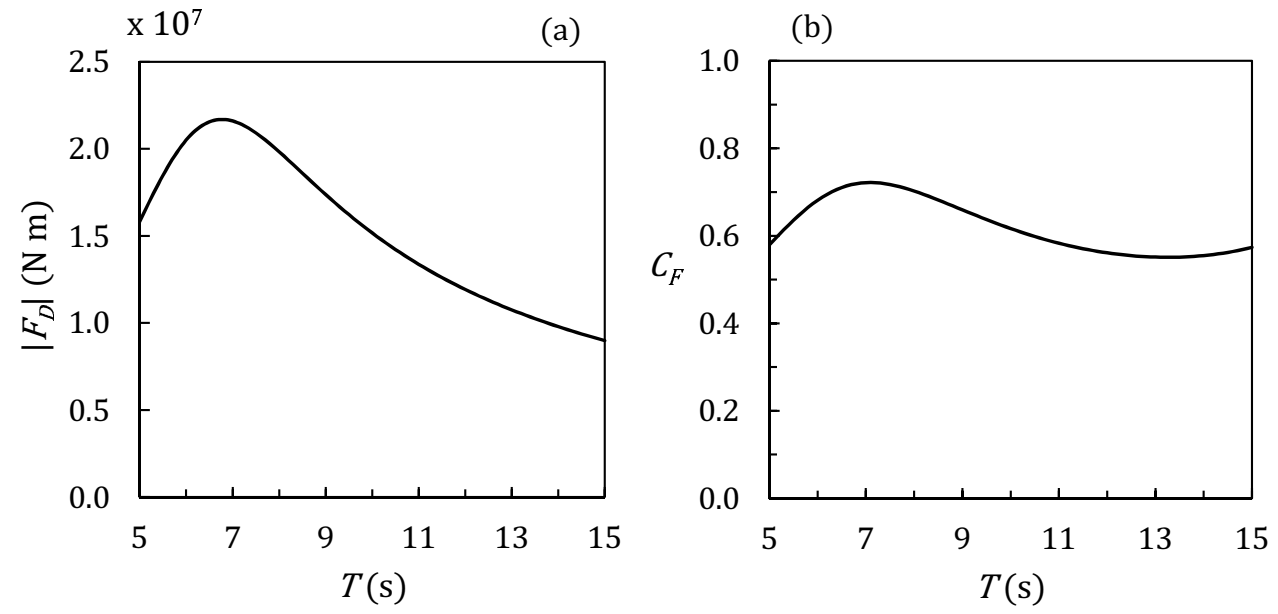

Figure 7: (a) Magnitude of the exciting torque and (b) capture factor versus period of the incident waves for a flap layout similar to the Oyster 800 WEC designed by Aquamarine Power. Parameters are $A=1 \mathrm{~m}, w=26 \mathrm{~m}, h=13 \mathrm{~m}, c=4 \mathrm{~m}$. The semi-analytical model of Renzi and Dias [11] for a single flap in the open ocean has been used.

not necessary to enhance power output. As a matter of fact, the natural period

$$
T_{0}=2 \pi \sqrt{\frac{I+\mu}{C}}
$$

of a typical Oyster configuration is much larger than the torque peak period $T_{\text {peak }}$, usually being $T_{0} \simeq 20 \mathrm{~s}[9,25]$. Now recall that the natural period of a floating body is the period at which it would oscillate indefinitely, when released from a perturbed configuration, in the absence of incident waves and damping [16]. When the incident wave period equals the natural period, i.e. $T=T_{0}=2 \pi / \omega_{0}$, Eq. (10) with $\omega=\omega_{0}=\sqrt{C /(I+\mu)}$ yields $\nu_{p t o}=\nu$ and the body is tuned to resonate with amplitude

$$
\left|\Theta_{0}\right|=\frac{\left|F_{D}\right|}{2 \omega_{0} \nu}
$$

from Eq. (8). However, for Oyster the requirements to have both maximum torque and tuning are incompatible, since the peak torque is attained at $T_{\text {peak }} \ll T_{0}$. As already shown by [7], to decrease the natural period $T_{0}$ (13) one could reduce the total inertia of the flap, e.g. by making it smaller. In turn, this not only will decrease the wave torque $F_{D}$, but also the radiation damping $\nu$. However, while decreasing the size of the flap, the radiation 
damping $\nu$ decreases much faster on average than the other hydrodynamic parameters, as shown in figure 8 (see also figure 7 of [11]). As a consequence, reducing the size of the flap would ultimately increase the pitching amplitude $\left|\Theta_{0}\right|$ (14). Therefore, a smaller flap tuned to resonance would have to move to extremely large amplitude $\left|\Theta_{0}\right|$ (14) to capture more power. This is not possible as the flap motion is limited by the seabed. Hence the flap would not produce the predicted power, i.e. it would not be optimal ([7] and [23] proposed a similar reasoning). This explains why Oyster is not tuned to resonance. In other words, rather than exploiting unrealistic resonant tuning [7], Oyster's working principle relies on the exploitation of the peak wave torque which by design occurs at periods close to the mean energetic periods in normal operational conditions. So far, we have highlighted the peculiar diffractive behaviour of Oyster, which makes its working principle very different from that of a point-absorber device. In the next section we will show that the intrinsically 3D dynamics of Oyster also rules out the applicability of simplified 2D models to estimate its efficiency.

\subsection{Conquering the third dimension}

Oyster's large width could tempt to adopt a simplifying 2D representation for evaluating its performance, by assuming that the device is infinitely wide. Indeed, Oyster's law of motion (8) looks formally equivalent to that of the 2D terminator absorber model in Chapter 8.9 of [16], for which the hydrodynamic efficiency (i.e. the 2D counterpart of the capture factor, see [10]) satisfies

$$
E_{f} \leq 1 / 2,
$$

assuming the body is symmetric about its vertical axis. However, such similarity is only formal: the hydrodynamic coefficients $F_{D}, \mu$ and $\nu$ in (8) and (11) contain information on the three-dimensional interaction occurring between Oyster and the waves. Hence they differ largely from their 2D counterparts in the terminator absorber model of [16]. As a result, (15) does not apply to Oyster. This is clearly shown in figure 7(b) for the Oyster 800, where $\max C_{F} \simeq 0.72$ in violation of Eq. (15). The reason why Oyster's capture factor can be larger than the theoretical 2D limit (15) lies mainly in the exciting torque $F_{D}$. Figure 9 shows a qualitative scheme of the diffraction pattern of a fixed flap in incident waves, for both the $2 \mathrm{D}$ and $3 \mathrm{D}$ cases. In 2D the incident wave field is completely reflected back in the opposite direction, while water is at rest on the lee side of the flap. In 3D, on the other hand, the 

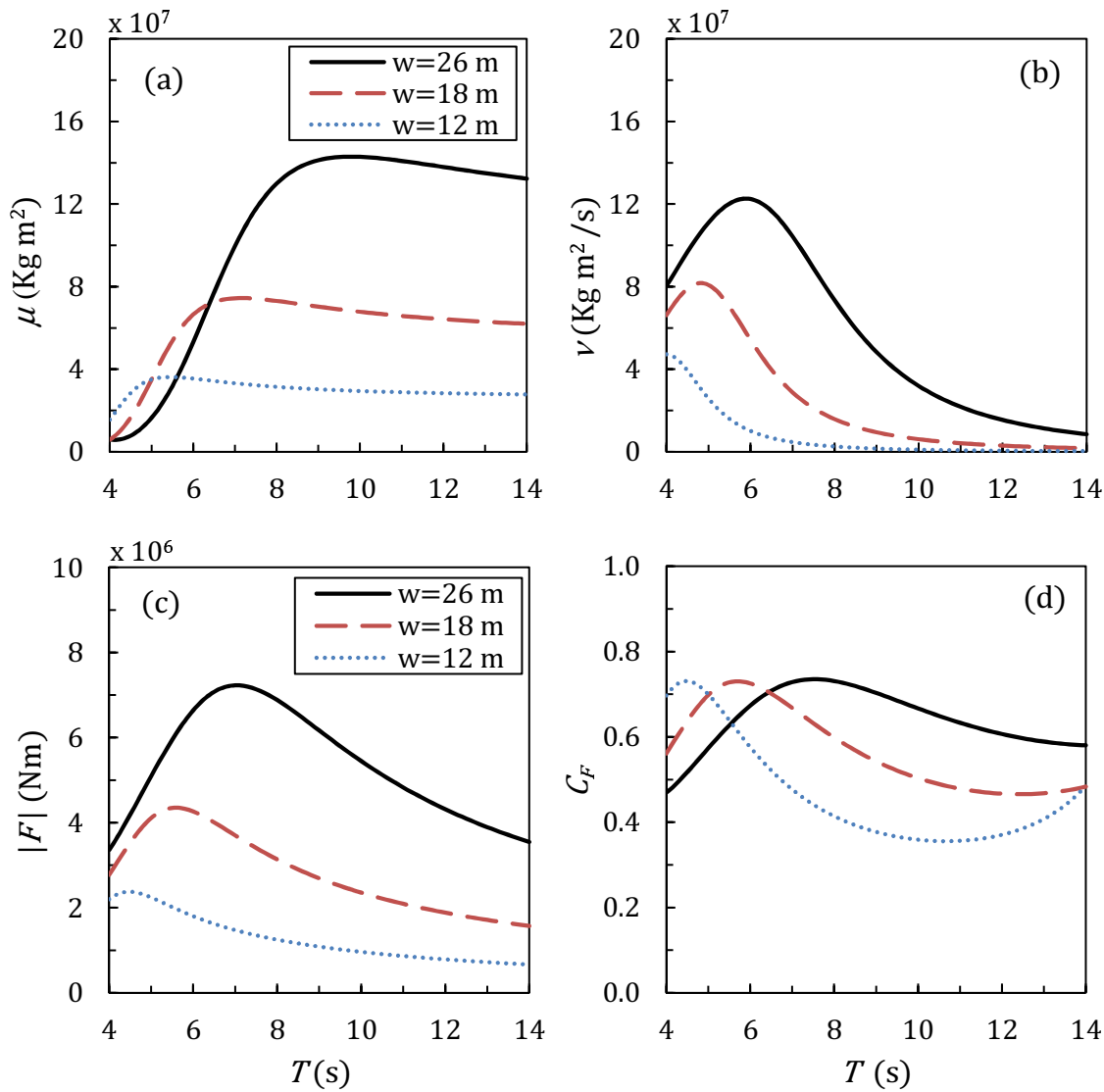

Figure 8: Behaviour of (a) added inertia torque, (b) radiation damping, (c) magnitude of the exciting torque and (d) capture factor versus the period of the incident wave. Parameters are $A=0.3 \mathrm{~m}, h=10.9 \mathrm{~m}, c=1.5 \mathrm{~m}$. Three different flap width have been considered. The figure is reproduced from [11]. Note that the radiation damping is (on average) the most sensitive hydrodynamic quantity to a variation of the flap width. 


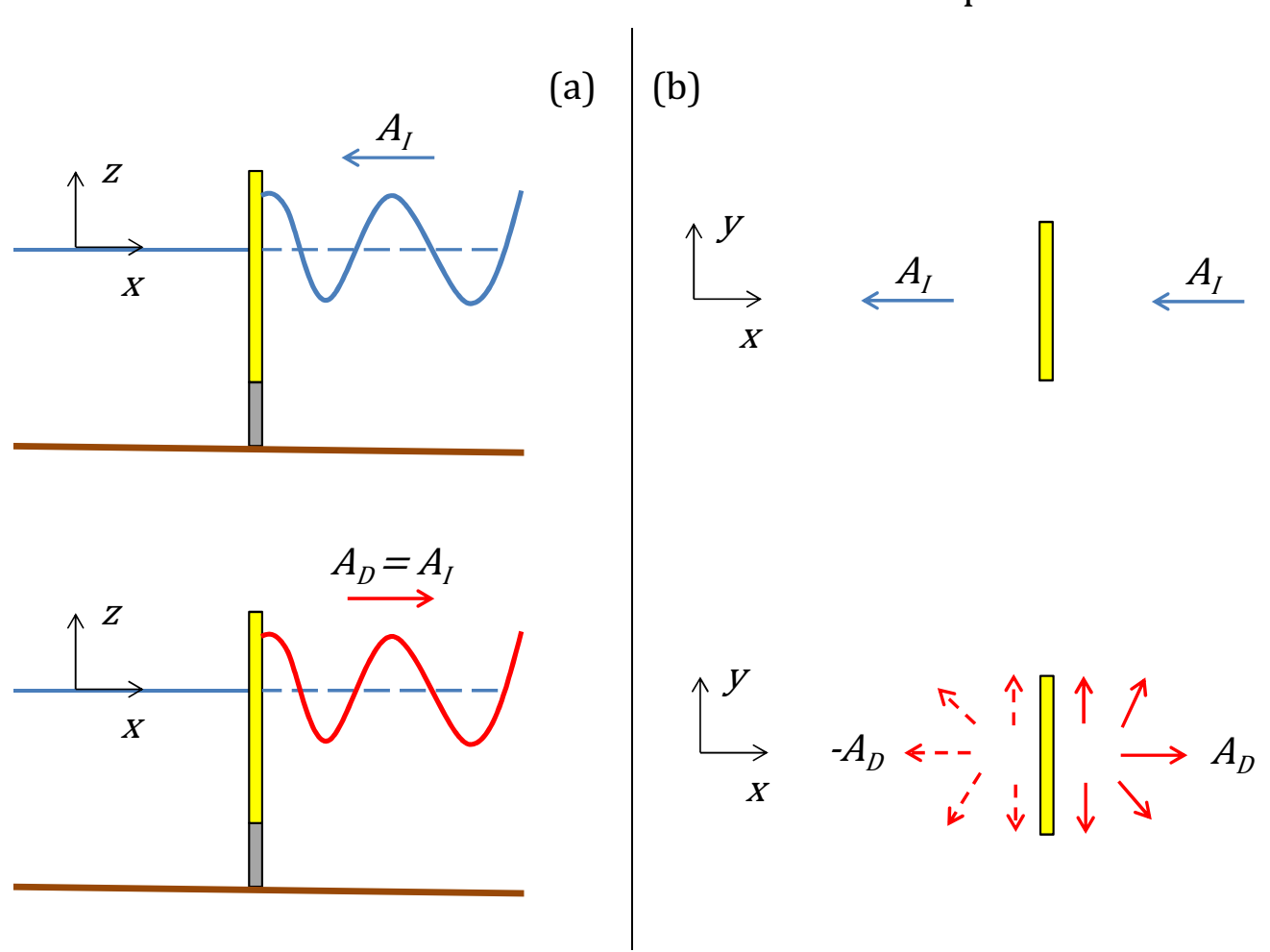

Figure 9: Qualitative scheme of the diffraction mechanism of a fixed flap, for (a): a 2D terminator (section), (b): a 3D flap-type converter (plan view). In (a) the diffracted wave is equal and opposite to the incident wave because of complete reflection. A standingwave field is generated on the weather side of the flap, while water is at rest on the lee side. In (b) antisymmetric diffracted waves travel in all directions, including tangentially to the flap. The presence of antisymmetric transverse waves along the flap can trigger near-resonant phenomena which enhance the exciting torque acting on the converter with respect to the $2 \mathrm{D}$ scenario (see [13] for a quantitative analysis of the phenomenon). 
diffracted waves are antisymmetric with respect to the flap longitudinal axis and propagate in all directions, including tangentially along the flap $[11,12]$. The presence of antisymmetric transverse waves along the flap can trigger a mechanism termed "near resonance" by Renzi et al. [13]. Near resonance amplifies the exciting torque acting on the converter and hence the capture factor (11), which raises well above the $2 \mathrm{D}$ theoretical limit (15). It is important to emphasise that near resonance is a phenomenon arising because of diffraction and has nothing to do with the body resonance discussed in Section 4.

\section{Conclusion: the flap-type absorber model}

Failure of existing point-absorber and 2D terminator theories to accurately reproduce the dynamics of Oyster has urged the introduction of a new mathematical reference model for OWSCs, that we now term "flap-type absorber". The flap-type absorber model describes a large device of negligible thickness which extracts energy by pitching about a horizontal axis parallel to the ocean bottom. Mathematically, given the incident wavenumber $k$, the flap width $w$ is such that $k w=O(1)$, while the thickness $a$ satisfies $a / w \ll 1$. As shown in Section 4, the mechanism of power absorption by a flap-type absorber is diffraction-driven. That is, the flap-type absorber extracts energy driven by the large wave torque resulting from the modification of the incident wave field, induced by the obstructing action of the flap. This concept is fundamentally innovative with respect to other WEC concepts, like floating buoys [6] and attenuators [22], which are based on the point-absorber criterion that a good wave energy absorber must be a good wave radiator. In this paper, we have shown that this early approach does not apply to an OWSC like Oyster, which is found to have the ability to absorb significant amount of energy at short periods in small-amplitude oscillations, without the need of unrealistic tuning or motion constraints usually adopted by point- or lineabsorbers. Finally, we have also shown that simplified 2D terminator models can significantly underestimate the power capture of a flap-type absorber. In conclusion, the behaviour of OWSCs in general, and Oyster in particular, is very different from that of a point absorber or a two-dimensional terminator device, as summarised in table 1.

In this paper, we have used the mathematical formulation of the flaptype absorber to explain how Oyster works and why modelling its dynamics requires a different mathematical framework with respect to former WEC 


\begin{tabular}{ccccc} 
Model & Dimension & Geometry & Mechanism & Ref. \\
\hline Point absorber & $3 \mathrm{D}$ & $k r \ll 1$ & Resonant & {$[21]$} \\
Terminator & 2D & $k w \gg 1$ & Resonant & {$[16]$} \\
Flap-type absorber & $3 \mathrm{D}$ & $\begin{array}{c}k w=O(1) \\
a / w \ll 1\end{array}$ & Torque driven & {$[11,12]$} \\
\hline
\end{tabular}

Table 1: Summary of properties for the point-absorber, terminator and flap-absorber models discussed here. From left to right, dimensions, geometrical characteristics of the device and power extraction mechanisms are compared, providing the relevant bibliographic references. $k$ is the wavenumber of the incident wave, $r$ the characteristic dimension of the point absorber, $w$ denotes the width of the device and $a$ the thickness.

concepts.

E.R. and F.D. were funded by Science Foundation Ireland (SFI) under the research project "High-end computational modelling for wave energy systems".

\section{References}

[1] J. Grue, E. Palm, Reflection of surface waves by submerged cylinders, Applied Ocean Research 6(1) (1984) 54-60.

[2] J. Grue, E. Palm, Wave radiation and wave diffraction from a submerged body in a uniform current, Journal of Fluid Mechanics 151 (1985) 257278.

[3] E. Palm, Nonlinear wave reflection from a submerged circular cylinder, Journal of Fluid Mechanics 233 (1991) 49-63.

[4] J. Nossen, J. Grue, E. Palm, Wave forces on three-dimensional floating bodies with small forward speed, Journal of Fluid Mechanics 227 (1991) $135-160$.

[5] G. Thomas, The theory behind the conversion of ocean wave energy: a review, in: Ocean Wave Energy (J. Cruz ed.), Springer, Germany, 2008.

[6] J. Falnes, J. Hals, Heaving buoys, point absorbers and arrays, Philosophical Transaction of the Royal Society A 370 (2012) 246-277. 
[7] T. Whittaker, M. Folley, Nearshore oscillating wave surge converters and the development of Oyster, Philosophical Transaction of the Royal Society A 370 (2012) 345-364.

[8] S. Crowley, R. Porter, D.V. Evans, A submerged cylinder wave energy converter, Journal of Fluid Mechanics 716 (2013) 566-596.

[9] E. Renzi, F. Dias, Resonant behaviour of an oscillating wave energy converter in a channel, Journal of Fluid Mechanics 701 (2012) 482-510.

[10] E. Renzi, F. Dias, Relations for a periodic array of oscillating wave energy converters, Applied Ocean Research 39 (2013) 31-39.

[11] E. Renzi, F. Dias, Hydrodynamics of the oscillating wave surge converter in the open ocean, European Journal of Mechanics B/Fluids 41 (2013) $1-10$.

[12] E. Renzi, F. Dias, Mathematical modelling of a flap-type wave energy converter, in: Proceedings of the 32nd International Conference on Ocean, Offshore and Arctic Engineering, OMAE2013, Nantes, France, 2013.

[13] E. Renzi, A. Abdolali, G. Bellotti, F. Dias, Wave-power absorption from a finite array of Oscillating Wave Surge Converters, Renewable Energy 63 (2014) 55-68.

[14] D. Sarkar, E. Renzi, F. Dias, Wave power extraction by an oscillating wave surge converter in random sea, in: Proceedings of the 32nd International Conference on Ocean, Offshore and Arctic Engineering, OMAE2013, Nantes, France, 2013.

[15] P. Schmitt, S. Bourdier, D. Sarkar, E. Renzi, F. Dias, K. Doherty, T. Whittaker, J. van't Hoff, Hydrodynamic Loading on a Bottom Hinged Oscillating Wave Surge Converter, in: Proceedings of the Twenty-second International Offshore and Polar Engineering Conference, ISOPE, Rhodes, Greece, 2012, pp. 550-557.

[16] C.C. Mei, M. Stiassnie, D.K.-P. Yue, Theory and application of ocean surface waves, World Scientific, USA, 2005. 
[17] A. Rafiee, F. Dias, Numerical simulation of wave impact on an oscillating wave surge converter, in: Proceedings of the 2nd International Conference on Violent Flows, Nantes, France, 2012, pp. 157-163.

[18] Y. Wei, A. Rafiee, F. Dias, On the viscous effects in the interaction of water waves with an oscillating wave surge converter, in: Proceedings of the 10th European Wave and Tidal Energy Conference, EWTEC13, Aalborg, Denmark, 2013.

[19] Y. Wei, A. Rafiee, B. Elsaesser, F. Dias, Numerical simulation of an oscillating wave surge converter, in: Proceedings of the 32nd International Conference on Ocean, Offshore and Arctic Engineering, OMAE2013, Nantes, France, 2013.

[20] A. Henry, A. Rafiee, P. Schmitt, F. Dias. The Characteristics of Wave Impacts on an Oscillating Wave Surge Converter, in: Proceedings of the Twenty-third International Offshore and Polar Engineering Conference, ISOPE, Anchorage, Alaska, USA, 2013, pp. 566-573.

[21] J. Falnes. Ocean Waves and Oscillating Systems, Cambridge University Press, U.K., 2002.

[22] R. Yemm, D. Pizer, C. Retzler, R. Henderson, Pelamis: experience from concept to connection, Philosophical Transaction of the Royal Society A 370 (2012) 365-380.

[23] M. Folley, T.J.T Whittaker, A. Henry, The effect of water depth on the performance of a small surging wave energy converter, Ocean Engineering 34 (2007) 1265-1274.

[24] S. Gallagher, R. Tiron, F. Dias, A detailed investigation of the nearshore wave climate and the nearshore wave energy resource on the west coast of Ireland, in: Proceedings of the 32nd International Conference on Ocean, Offshore and Arctic Engineering, OMAE2013, Nantes, France, 2013.

[25] H. Henry, K. Doherty, L. Cameron, T. Whittaker, R. Doherty, Advances in the design of the Oyster wave energy converter. In RINA Marine and Offshore Renewable Energy, London, UK, 2010. 